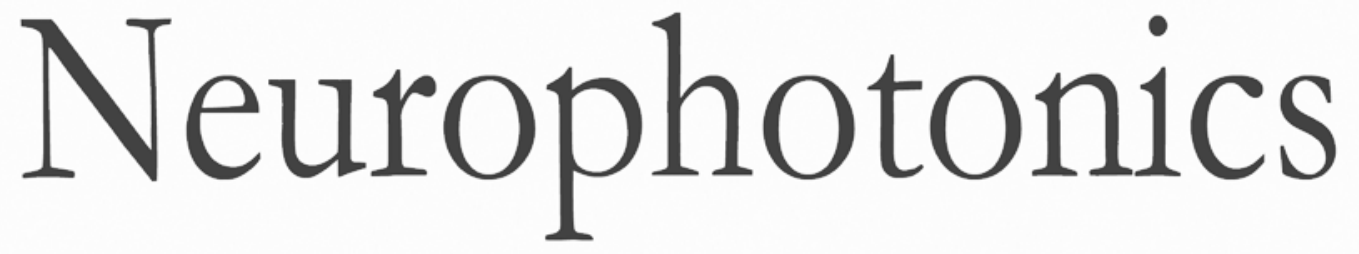

\title{
Label-free photoacoustic tomography of whole mouse brain structures ex vivo
}

Lei Li

Jun Xia

Guo Li

Alejandro Garcia-Uribe

Qiwei Sheng

Mark A. Anastasio

Lihong V. Wang 


\title{
Label-free photoacoustic tomography of whole mouse brain structures ex vivo
}

\author{
Lei Li, ${ }^{a, b}$ Jun Xia, ${ }^{c}$ Guo Li, ${ }^{b}$ Alejandro Garcia-Uribe, ${ }^{b}$ Qiwei Sheng, ${ }^{b}$ Mark A. Anastasio, ${ }^{b}$ and Lihong V. Wang ${ }^{a, b, \star}$ \\ ${ }^{a}$ Washington University in St. Louis, Department of Electrical and System Engineering, One Brookings Drive, St. Louis, Missouri 63130, \\ United States \\ ${ }^{b}$ Washington University in St. Louis, Department of Biomedical Engineering, One Brookings Drive, St. Louis, Missouri 63130, United States \\ 'University at Buffalo, The State University of New York, Department of Biomedical Engineering, 332 Bonner Hall, Buffalo, New York 14260, \\ United States
}

\begin{abstract}
Capitalizing on endogenous hemoglobin contrast, photoacoustic-computed tomography (PACT), a deep-tissue high-resolution imaging modality, has drawn increasing interest in neuroimaging. However, most existing studies are limited to functional imaging on the cortical surface and the deep brain structural imaging capability of PACT has never been demonstrated. Here, we explicitly studied the limiting factors of deep brain PACT imaging. We found that the skull distorted the acoustic signal and blood suppressed the structural contrast from other chromophores. When the two effects are mitigated, PACT can potentially provide high-resolution label-free imaging of structures in the entire mouse brain. With $100-\mu \mathrm{m}$ in-plane resolution, we can clearly identify major structures of the brain, which complements magnetic resonance microscopy for imaging small-animal brain structures. Spectral PACT studies indicate that structural contrasts mainly originate from cytochrome distribution and that the presence of lipid sharpens the image contrast; brain histology results provide further validation. The feasibility of imaging the structure of the brain in vivo is also discussed. Our results demonstrate that PACT is a promising modality for both structural and functional brain imaging. (๑) 2016 Society of Photo-Optical Instrumentation Engineers (SPIE) [DOI: 10.1117/1.NPh.3.3.035001]
\end{abstract}

Keywords: photoacoustic-computed tomography; brain structural imaging; deep brain imaging; label free; spectral imaging

Paper 16030PR received May 19, 2016; accepted for publication Jul. 6, 2016; published online Jul. 26, 2016.

\section{Introduction}

Revealing how the brain works is a grand challenge worth our every effort. It will provide the key to understand and treat neurological diseases. ${ }^{1}$ Over the last few decades, various functional and structural brain imaging techniques have been developed, and some can image both structures and functions using the same modality. For instance, magnetic resonance imaging (MRI) using the T1 and T2 contrasts can image the structure of the brain over a large volume. Using the diffuse tensor method, it can also reveal fractional anisotropy, three-dimensional (3-D) diffusivity, and connection information. Functional MRI can also be achieved based on the blood-oxygen-level dependent contrast. However, MRI works on time scales of minutes ${ }^{2,3}$ and requires a costly magnetic field to achieve high spatial resolution $(20-60 \mu \mathrm{m}) .^{4-6}$ Microscopic MRI of the mouse brain requires additional tissue staining and takes more than 1 day for data acquisition. ${ }^{7,8}$ Optical imaging techniques, such as confocal fluorescence microscopy and two-photon microscopy, are other powerful tools for both structural and functional brain imaging. ${ }^{9-12}$ Various bio-molecules, such as hemoglobin, cytochrome, melanin, and lipid, ${ }^{13-15}$ possess different optical properties, which provide contrast for structural brain imaging. Using endogenous and exogenous contrasts, light can also be used to monitor functional dynamics, such as hemodynamics and neuron activities. ${ }^{11,16,17}$ However, deep-tissue optical imaging at high spatial resolution is a major challenge because of tissue's strong optical scattering. Thus, most optical

*Address all correspondence to: Lihong V. Wang, E-mail: Ihwang@ wustl.edu imaging techniques can image only $1 \mathrm{~mm}$ deep into the brain. ${ }^{1}$

Fortunately, by converting photons into ultrasonic waves, which are orders of magnitude less scattered than light, photoacoustic (PA) tomography (PAT) can form images by detecting the pressure waves induced by the thermoelastic expansion of light-absorbing objects. ${ }^{18-21}$ PAT can break through the optical diffusion limit $(\sim 1 \mathrm{~mm}$ depth) by capitalizing on the lowacoustic scattering in tissue. Moreover, the scalability of PAT provides an unprecedented opportunity to bridge the gap between microscopic and macroscopic images. PAT is capable of anatomical, functional, molecular, and metabolic imaging of small animals, with highly scalable spatial resolution and penetration depth. ${ }^{21-23}$ Photoacoustic-computed tomography (PACT), a major implementation of PAT, provides fast data acquisition, submillimeter resolution, and deep penetration beyond the optical diffusion limit. ${ }^{22-25}$ Noninvasive, label-free, and functional PACT of the brains of small animals has been demonstrated by accurately mapping brain lesions and cerebral hemodynamics. ${ }^{26}$ Also, Functional imaging of brain metabolism and hemodynamic changes has been done by PACT. ${ }^{27}$

Here, for the first time, we experimentally demonstrate that PACT is able to provide label-free imaging of structures in the whole mouse brain. PACT complements magnetic resonance microscopy (MRM) for imaging small-animal brain structures by providing optical contrast. With $100-\mu \mathrm{m}$ lateral resolution and $400-\mu \mathrm{m}$ elevational resolution, most deep brain anatomical structures have been clearly resolved through the entire brain. 
We also found by spectral analysis that the structural contrast comes mainly from cytochrome and lipid.

\section{Materials and Methods}

\subsection{Full-Ring Photoacoustic-Computed Tomography System}

Figure 1 is a schematic of the PACT system setup. To provide illumination in the visible band (from 420 to $680 \mathrm{~nm}$ ), an optical parametric oscillator laser (BasiScan 120, Spectra-Physics) is pumped by an Nd:YAG laser (Brilliant B, Quantel) with a third-harmonic generator $(355 \mathrm{~nm})$. The laser pulse width is $6 \mathrm{~ns}$, and the pulse repetition rate is $10 \mathrm{~Hz}$. The incident laser beam, reflected by a right-angle prism (PS912, Thorlabs), is homogenized through an engineered diffuser (EDC-5, RPC Photonics) to provide uniform illumination over the mouse brain. The maximum laser fluence on the scalp is $\sim 6 \mathrm{~mJ} / \mathrm{cm}^{2}$, which is well below the American National Standards Institute safety limit $\left(20 \mathrm{~mJ} / \mathrm{cm}^{2}\right.$ in the visible spectral region). The photoacoustic signals are detected by a $5-\mathrm{cm}$ diameter fullring ultrasonic transducer array (Imasonic Inc.) with 512 elements, $5-\mathrm{MHz}$ central frequency, and more than $80 \%$ one-way bandwidth. Each element (10-mm high, $0.3 \mathrm{~mm}$ in pitch, and $0.1-\mathrm{mm}$ interelement spacing) is cylindrically focused to produce an axial focal distance of $19.8 \mathrm{~mm}$ (acoustic NA of 0.25 ). The combined foci of all elements form a uniform imaging region of 20-mm diameter and $1-\mathrm{mm}$ thickness. ${ }^{25}$ Within this region, the radial resolution is $100 \mu \mathrm{m}$ and the tangential resolution is 100 to $250 \mu \mathrm{m} .{ }^{28}$ The data acquisition system has 64 channels with eight-fold multiplexing. After a complete data acquisition from all 512 elements, the raw data are used to reconstruct an image based on the universal back-projection algorithm. ${ }^{29}$ Due to the bandwidth limit of the detectors, the reconstructed images manifest bipolar contrast. For images selected for quantitative studies, to mitigate errors (the bipolar effect) in reconstructed images, we applied a joint reconstruction method, ${ }^{30}$ where iterative reconstruction with a nonnegativity constraint was employed to solve the minimization problem. This approach seeks to refine the measured transducer responses during the process of reconstructing the sought-after absorbed optical energy density. 3-D images can be obtained by scanning the sample along the elevational direction $(z$-axis direction in

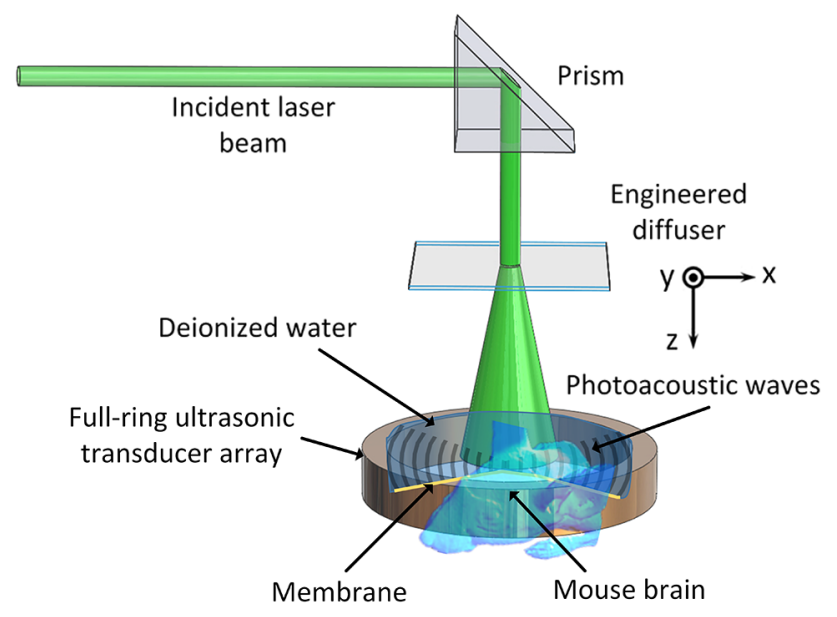

Fig. 1 Schematic of full-ring PACT system.
Fig. 1). The imaging speed of the full-ring PACT system is $1.6 \mathrm{~s}$ per frame (per cross-section).

\subsection{Label-Free Photoacoustic Imaging}

Based on the photoacoustic effect, if all absorbed optical energy is converted into heat, the initial pressure is given by ${ }^{31}$

$p_{0}=\Gamma \mu_{a} F$,

where $F$ is the optical fluence $\left(\mathrm{J} / \mathrm{cm}^{2}\right), \mu_{a}$ is the optical absorption coefficient $\left(\mathrm{cm}^{-1}\right)$, and $\Gamma$ is the Grueneisen parameter. It follows from Eq. (1) that PAT is exquisitely sensitive to optical absorption. In fact, among all optical imaging modalities, PAT has the highest sensitivity to optical absorption because it converts a small change in the optical absorption coefficient to an equal fractional change in the ultrasound signal, tantamount to a relative sensitivity of $100 \% .^{18}$

In principle, PAT can be used to image and quantify the concentration of any molecule, based on its absorption spectrum. For instance, PAT has been used to image oxy- and deoxyhemoglobin, ${ }^{21,32-34}$ melanin, ${ }^{35,36}$ water, ${ }^{37,38}$ lipids, ${ }^{39-41}$ DNA and RNA $^{42-44}$ and cytochromes. ${ }^{45,46}$ Figure 2(a) indicates that hemoglobin is the most optically absorbing contrast for wavelengths below $1000 \mathrm{~nm}$. In the visible spectral region, its absorption coefficient can exceed that of other chromosphores by more than two orders of magnitude. While hemoglobin allows acquisition of stunning vascular images, it also obscures PA signals from other endogenous chromosphores, such as cytochromes and lipid.

The in vivo performance of label-free PACT was tested in a nude mouse brain (Hsd: ND4, Harlan, 2-month-old, 20 to $24 \mathrm{~g}$ ). All experimental procedures were carried out in conformity with laboratory animal protocols approved by the Animal Studies Committee at Washington University in St. Louis. Throughout the experiment, the mouse was maintained under anesthesia with $1.5 \%$ vaporized isoflurane. Before imaging, the hair on the mouse's head was removed with a depilatory. The mouse was then taped to a lab-made motorized animal holder, which was then mounted to the PACT system. Instead of being fully immersed in water, the animal was supported from below, with the head being covered by a flexible membrane. As shown in Fig. 2(b), with 600-nm light illumination, a PACT image acquired on the brain surface shows rich cortical vasculature. Signals from major vessels, such the superior sagittal sinus, are so strong that their shadows even appear in the deep brain image [Fig. 2(c)]. Nevertheless, the skull strongly attenuates and distorts the acoustic signal. Consequently, even though different brain structures have different cytochrome and lipid concentrations, which might provide us with structural contrast, we still cannot differentiate them in the deep brain image.

\subsection{Saline-Perfused Mouse Brain Imaging by Photoacoustic-Computed Tomography}

From Fig. 2(a), we can see that if hemoglobin is removed from tissue, cytochrome becomes the dominant absorber in the visible region. In a mouse brain, by dry weight, gray matter has 55\% protein and $33 \%$ lipid, white matter has $55 \%$ lipid and $40 \%$ protein, and myelin has $70 \%$ lipid and $29 \%$ protein. $^{49}$ Thus, the difference in protein/lipid concentrations can be utilized to differentiate the brain structures. To remove the hemoglobin, we used saline perfusion. 

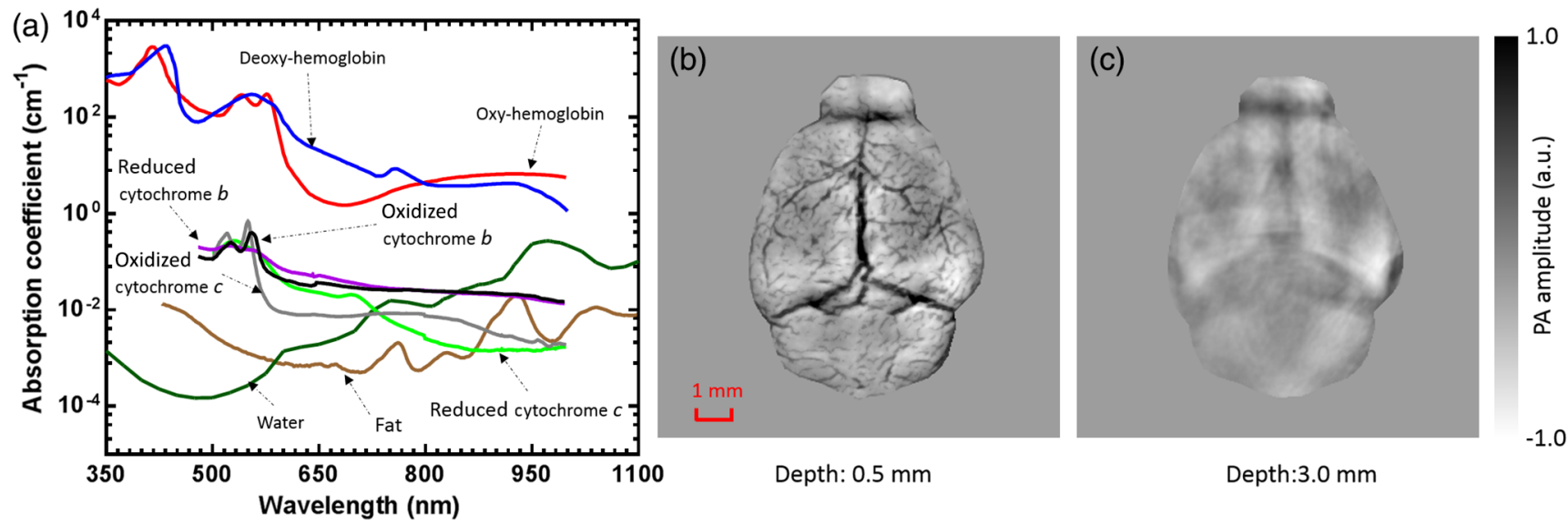

Fig. 2 Label-free PA imaging. (a) Absorption coefficient spectra of endogenous tissue chromophores at typical concentrations in the human body (adapted from Refs. 47 and 48). (b) In vivo imaging of the mouse brain cortex through the intact skull (imaging depth: $0.5 \mathrm{~mm}$ beneath the skull surface). (c) In vivo imaging of the deep mouse brain (imaging depth: $3.0 \mathrm{~mm}$ beneath the skull surface).

Saline-perfused mice were prepared by the Hope Center Animal Surgery Core at Washington University, following the standard mouse transcardial perfusion protocol. Blood was removed from the body tissues by pumping phosphate buffered saline into the left ventricle and draining the blood from the aorta, which was cut with scissors. After 4 to $5 \mathrm{~min}$, the fluid exiting the aorta was clear and the liver had turned white. At this point most of the blood in the body had been replaced with saline. We then dissected the head and placed it into $10 \%$ paraformaldehyde (PFA) solution for preservation. After $24 \mathrm{~h}$ of fixation in $10 \%$ PFA, we embedded the head in 3\% agar gel for imaging.

\section{Results}

\subsection{Photoacoustic-Computed Tomography of Mouse Brain Structures}

Saline-perfused mouse heads with skull intact were imaged with 600-nm laser illumination. Video 1(a) [Fig. 3(a)] shows cross-sectional images at different depths. Without the dominant hemoglobin absorption, this image looks totally different from Fig. 2(b). However, we still cannot clearly identify the structure of the brain, possibly due to light attenuation from the top skulls and acoustic distortion from the side skulls. To study the two effects, we first removed the top skulls. The resulting image [Fig. 3(b)] does not show much improvement, which indicates that light attenuation from the skull is negligible. We further removed the side skulls and acquired another image, which shows different brain structures with remarkable clarity [Fig. 3(c)]. To our knowledge, this is the first time that PAT has revealed deep structures of the brain in such detail based on endogenous contrast. Next, we removed the entire mouse brain from the rest of the skulls and acquired an image of the brain [Fig. 3(d)]. As can be seen by comparing Figs. 3(d) with $3(\mathrm{c})$, the image clarity was further improved by removing the mouth and nasal cavities, which interfere with acoustic propagation.

Figure 4(a) shows a label-free PACT image (acquired $2.8 \mathrm{~mm}$ below the brain surface) of another saline-perfused mouse brain, where the olfactory bulb was preserved. Images at other depths are shown in Video 2, from which we can see that PACT can image through the entire brain as deep as $5.0 \mathrm{~mm}$ in the absence of the side skulls. To better illustrate different regions, we segmented Fig. 4(a) based on the PA amplitude and labeled different segmentations [Fig. 4(b)]. For comparison, we chose one slice of a 3-D high-resolution (50- $\mu \mathrm{m}$ in-plane resolution) MRM image, with its structural segmentation superimposed as colored lines [Fig. 4(c)], ${ }^{50}$ as a gold standard. The mouse brain PACT
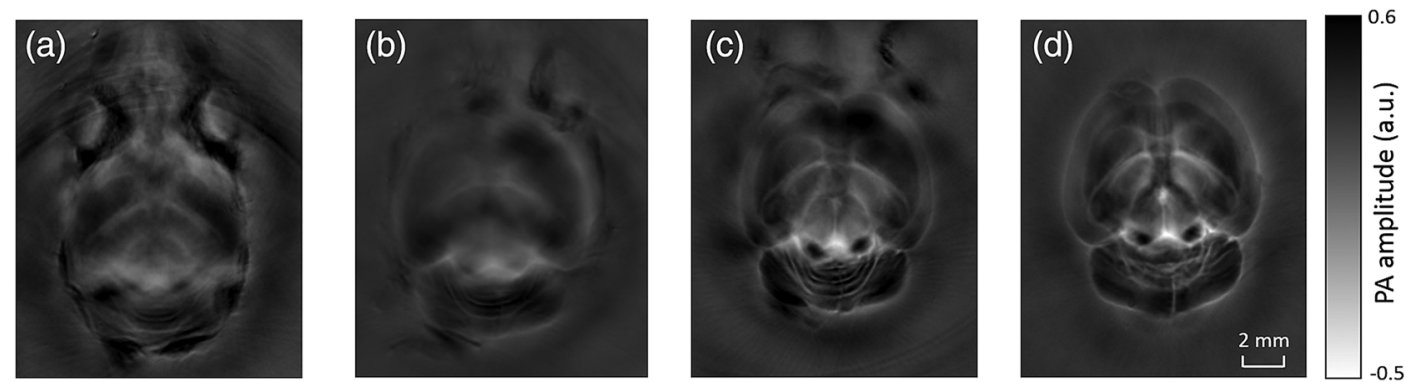

Fig. 3 PACT of the saline-perfused brain with and without the skull. (a) Image of in situ brain with skull intact at depth of $2.8 \mathrm{~mm}$ [Video 1(a)]. (b) Image of brain with top skulls removed at depth of $2.8 \mathrm{~mm}$ [Video 1(b)]. (c) Image of brain with top and side skulls removed at depth of $2.8 \mathrm{~mm}$ [Video 1(c)]. (d) Image of brain with all of the skulls removed at depth of $2.8 \mathrm{~mm}$ [Video 1(d), MOV, $414 \mathrm{~KB}$ ] [URL: http://dx.doi.org/10.1117/1.NPh.3.3.035001.1]. 

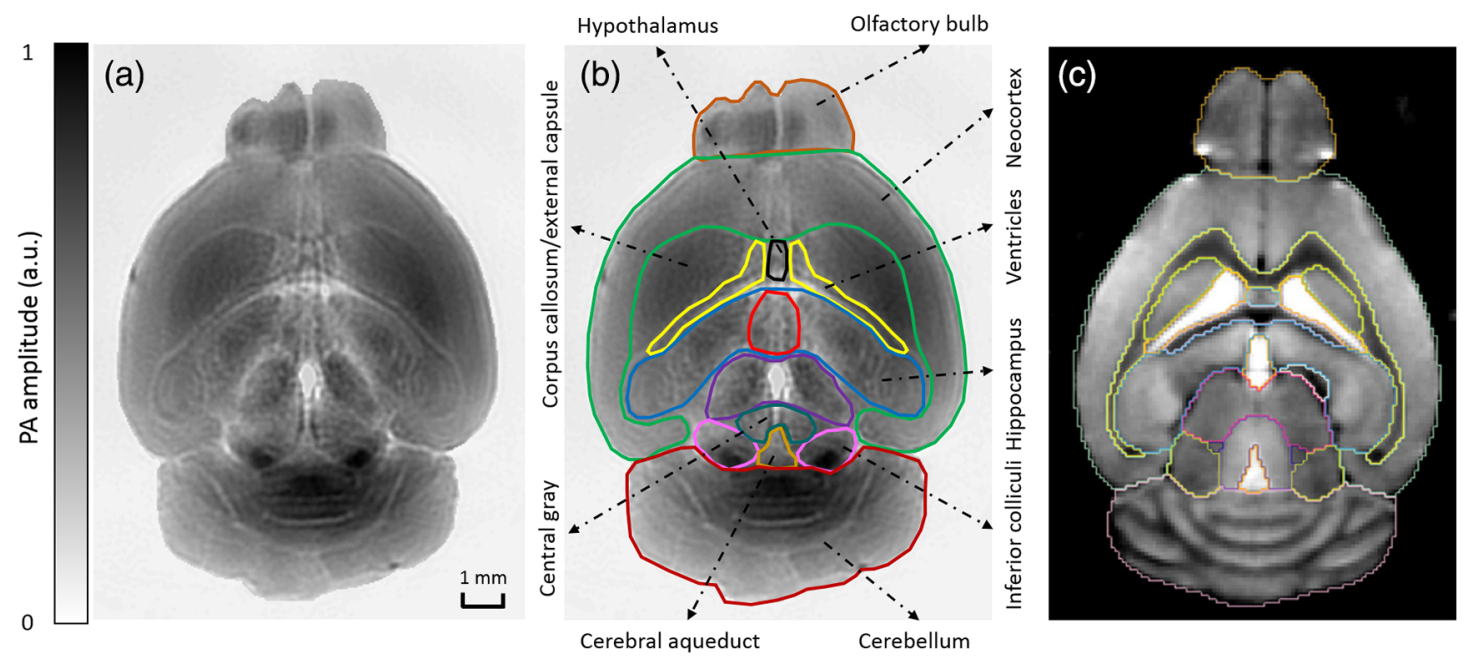

Fig. 4 Validation of the PACT image of the saline-perfused mouse brains without the skull. (a) Crosssectional PACT image of the mouse brain at $2.8-\mathrm{mm}$ depth, clearly resolving the structure of the brain. (b) Segmented and annotated image from Fig. 4(a). (c) One slice of a 3-D high-resolution MRM image with its structural segmentation superimposed as colored lines, chosen as a gold standard for validation of PACT ${ }^{50}$ (courtesy of Frontiers in Neuroscience) (Video 2, MOV, $135 \mathrm{~KB}$ ) [URL: http://dx.doi.org/10 .1117/1.NPh.3.3.035001.2].

image shows a nearly perfect match with the MRM image. Different brain structures are clearly identified, including the central gray, cerebellum, cerebral aqueduct, corpus callosum, hippocampus, hypothalamus, inferior colliculus, neocortex, olfactory bulb, and ventricles. To our knowledge, this is the first time that PACT has clearly shown deep structures of the brain with rich contrast among different brain tissues, with the brain intact and without any labeling. Such deep brain structures have not been shown by other photoacoustic imaging techniques or optical imaging modalities.

\subsection{Spectral Imaging}

To find the optimal wavelength for imaging brain structures and to verify the origin of contrasts, we varied the laser wavelength from 480 to $680 \mathrm{~nm}$ with a $20-\mathrm{nm}$ interval. Figure 5 shows the spectral PACT images of a mouse brain at one selected depth ( $2.8 \mathrm{~mm}$ below the brain surface), and Video 3 shows crosssection images at varied depths for each wavelength used (from 480 to $680 \mathrm{~nm}$ ). A quantitative spectral analysis is discussed in detail in Sec. 4.
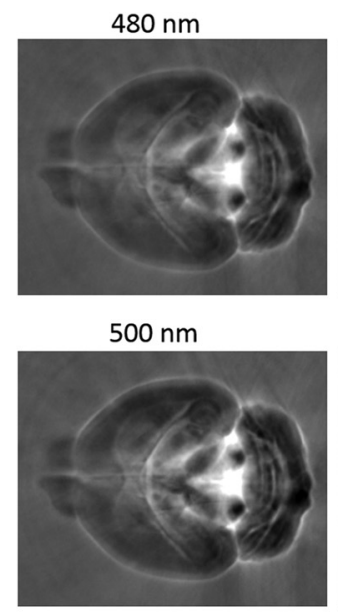

$520 \mathrm{~nm}$

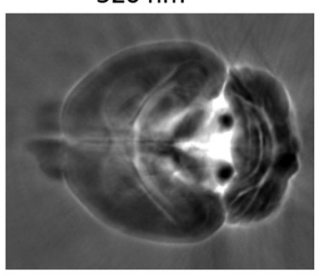

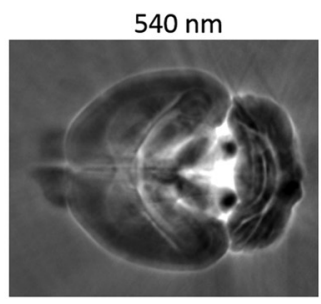

$560 \mathrm{~nm}$

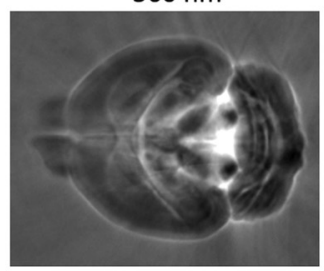

$580 \mathrm{~nm}$

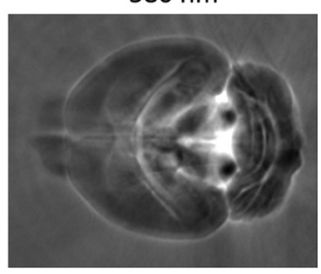

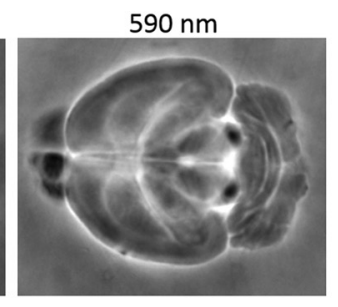

$600 \mathrm{~nm}$

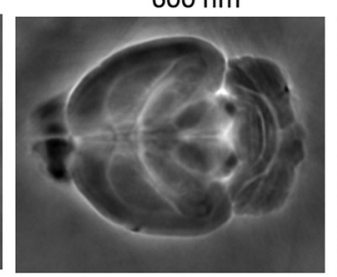

$620 \mathrm{~nm}$

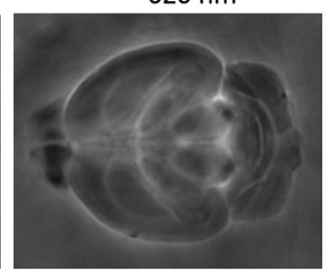

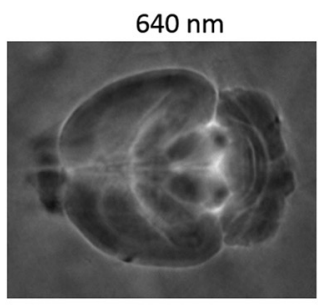

$660 \mathrm{~nm}$

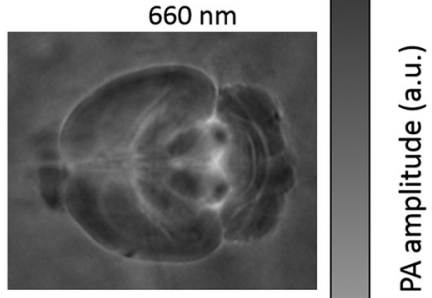

$680 \mathrm{~nm}$

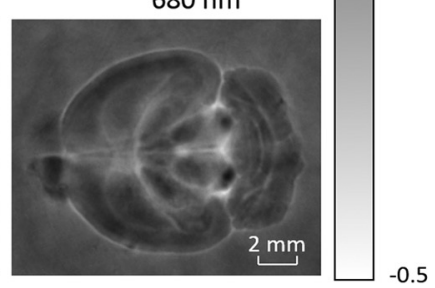

Fig. 5 Spectral PACT of a mouse brain at 2.8-mm depth (Video 3, MOV, 1.17 MB) [URL: http://dx.doi.org/ 10.1117/1.NPh.3.3.035001.3]. 


\section{Discussion}

After the removal of blood, the remaining dominant chromophores are nuclei (DNA and RNA), water, cytochrome, and lipid. DNA and RNA have a strong absorption in the ultraviolet band ( 240 to $280 \mathrm{~nm}$ ). However, in the visible light band (420 to $680 \mathrm{~nm}$ ), their absorption is negligible and is $10^{2}$ to $10^{3}$ times weaker than that of cytochrome. ${ }^{51}$ Water contents in various brain tissues are very similar ${ }^{52}$ and, thus, probably provide a relatively constant background in PACT images. Thus, the structural contrast should mainly come from cytochrome/lipid distribution. Each pixel in a single-wavelength PACT image represents a combined contribution from $M$ optical absorbers with known molar extinction coefficient spectra $a_{m}$ and unknown concentrations $c_{m}(m=1,2, \ldots, N)$. Then, the spectral decomposition equation can be expressed as

$\mu_{a}\left(\lambda_{n}\right)=\sum_{m=1}^{M} a_{m}\left(\lambda_{n}\right) c_{m}, \quad m=1,2, \ldots, N$,

where $\lambda_{n}$ is the $n$th wavelength used for PA excitation. Based on the known extinction coefficients, the concentrations of the optical absorbers can be quantified. In the following spectral analysis, we assume that differences between the Grüneisen parameters of different types of cytochromes are negligible, which was validated in a previous report. ${ }^{46}$

In mammals, the brain performs numerous computationally intensive tasks, such as information processing, perception, motion control, and learning, and, thus, consumes a large amount of energy in proportion to its volume. Mitochondria, the energy source, populate the cytoplasm of mammalian cells, including neurons, which rely on mitochondrial energy production for survival. ${ }^{53}$ The absorption sources of the mitochondria are mainly cytochromes $b$ and $c .^{46,54}$ Moreover, myelin has a high concentration of lipid, which has orders of magnitude weaker absorption than that of cytochrome over the spectral range of 480 to $680 \mathrm{~nm}$. Thus, the existence of myelin would further sharpen the contrast of cytochrome. Therefore, the distributions of chromophores map the brain with vivid contrast in PACT images.
Figure 6(a) shows the optical absorption contrast in one cross section ( $2.8 \mathrm{~mm}$ below the brain surface) based on the joint reconstruction approach. The spectral PA responses (normalized by the laser fluence) of the neocortex area encircled by the red line in Fig. 6(a) are plotted in Fig. 6(b) (labeled by circles). The neocortex consists of gray matter, or neuronal cell bodies and unmyelinated fibers. The high concentration of mitochondria in neuronal cell bodies might be responsible for the optical absorption. As the absorption sources in mitochondria are mainly cytochromes $b$ and $c,{ }^{46,54}$ the PA spectrum of the red outlined area was fitted according to Eq. (2) with a mixture of $65 \% \pm 12 \%$ (molar ratio, mean \pm standard error cytochrome $b$ and $35 \% \pm 9 \%$ cytochrome $c$, which is in agreement with the measured concentration of cytochrome in mitochondria. ${ }^{46}$ The squared 2-norm of the residual is 0.003 . The accuracy of spectral analysis, however, is subject to the possible presence of other neglected absorbing proteins (such as cytochrome p450, nitric-oxide synthases, and myeloperoxidase) with similar spectra. Absorption from other sources, such as water, flavoproteins, nicotinamide adenine dinucleotide, or other neglected hemeproteins, is orders of magnitude weaker than cytochrome, and was removed as a constant background during the fitting. A hematoxylin and eosin (H\&E) stained histology image [Fig. 6(c)] of a horizontal brain section is presented as a validation of the optical absorber, where pink represents the proteins in the cytoplasm and blue represents DNA/RNA in nuclei. In the H\&E stained histology image, the brain structure contrast from cytoplasm distribution matches with the absorption contrast-based PACT brain structure image, whereas the optical absorption from DNA/RNA is negligible under visible light illumination. This comparison between our PACT image and the H\&E stained histology image further validates that the structural contrast is mainly caused by proteins in cytoplasm.

To further confirm that structural contrast comes mainly from cytochrome and lipid, another area in the cerebellum [Fig. 7(a)] was segmented for spectral analysis. In a close-up image [Fig. 7(b)], white and black stripes correspond to cerebellar white matter and the granular layer, respectively. ${ }^{55,56}$ The cerebellar white matter, made up largely of myelinated nerve fibers,
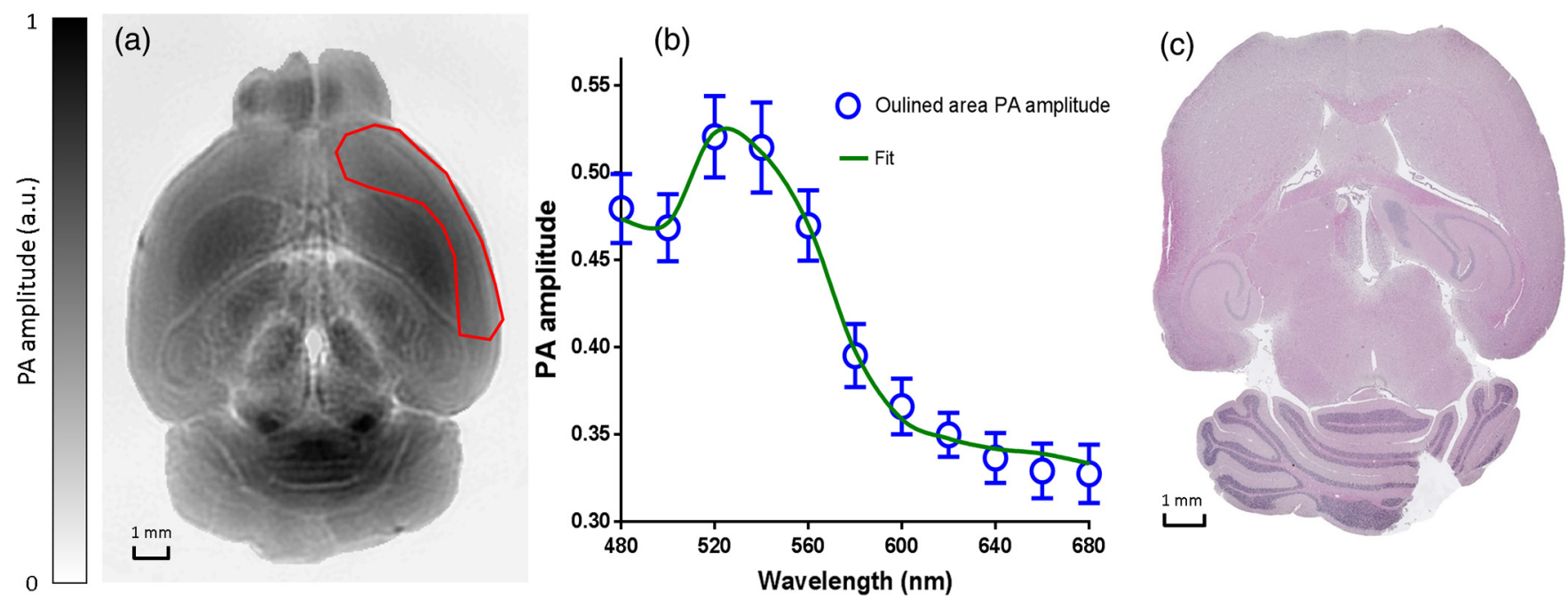

Fig. 6 Spectral analysis of the origin of optical absorption in the brain. (a) Cross-section imaged at 600$\mathrm{nm}$ wavelength at 2.8-mm depth as part of the 3-D brain imaging, with red outlined areas segmented for analysis. (b) Measured PA spectrum and least-squares fit with a mixture of cytochromes $b$ and $c$. (c) Histology image of one horizontal section slice stained with $\mathrm{H} \& \mathrm{E}$. 

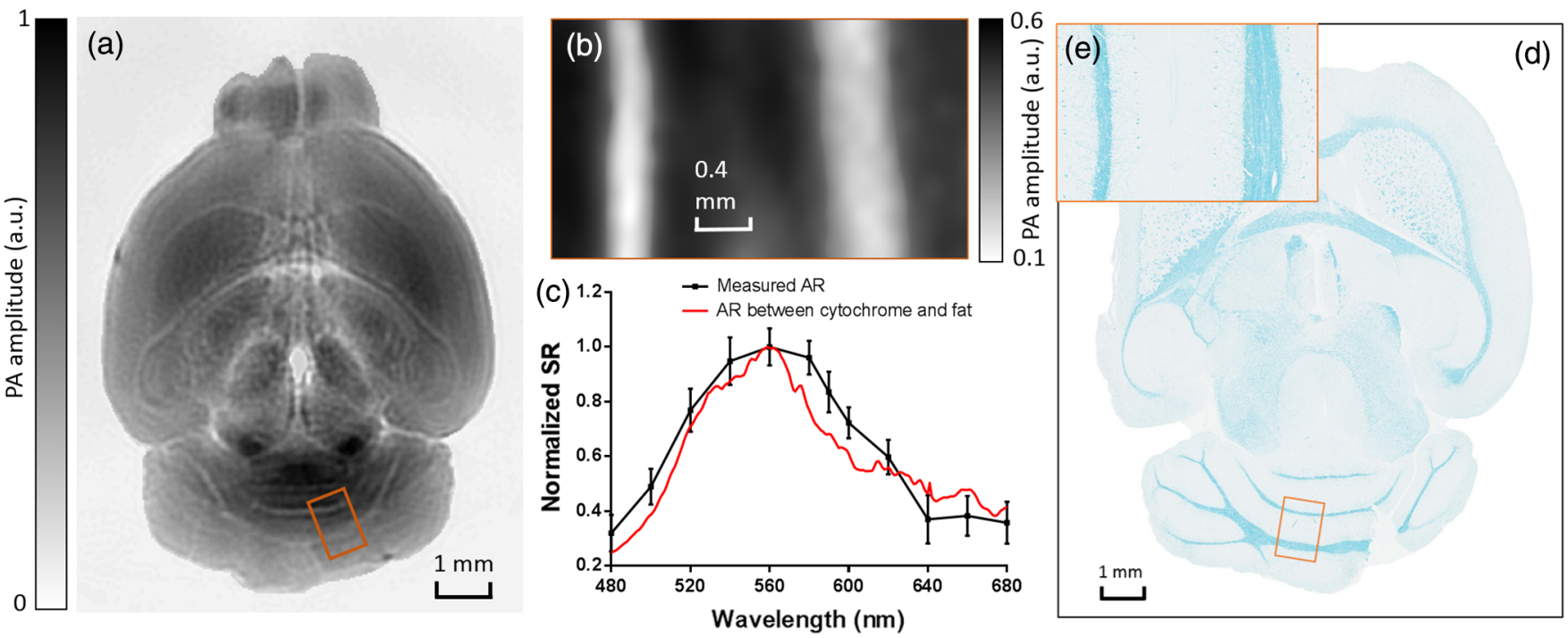

Fig. 7 Analysis of the origins of the contrast. (a) Cross-section imaged at 600-nm wavelength at 2.8-mm depth selected from the 3-D brain images, with brown outlined areas segmented for analysis. (b) Closeup of the region inside the brown square in Fig. 7(a). (c) Comparison of the measured AR between the granular cell layer and the cerebellar white matter and the AR between cytochrome and fat. (d) Histology image of one horizontal section slice stained with luxol fast blue. (e) Close-up of the region inside the brown square in Fig. 7(d).

has high concentration of lipid, and the granular layer has a high concentration of cytochrome. We calculated the absorption ratio (AR) between the granular cell layer and the cerebellar white matter as follows:

$\mathrm{AR}=\frac{\operatorname{mean}\left(\mathrm{PA}_{\mathrm{cwm}}\right)}{\text { mean }\left(\mathrm{PA}_{\mathrm{gcl}}\right)}$,

where $\mathrm{PA}_{\mathrm{cwm}}$ is the PA amplitude of the cerebellar white matter region and $\mathrm{PA}_{\mathrm{gcl}}$ is the $\mathrm{PA}$ amplitude of the granular layer region. We compare the result with the $\mathrm{AR}$ of cytochrome to lipid, which is obtained by taking the ratio point by point between the cytochrome absorption spectrum and the lipid absorption spectrum shown in Fig. 2(a). The two results match well with each other [Fig. 7(c)], which indicates that the contrast of the PACT image of the saline-perfused brain comes mainly from cytochrome and lipid. To confirm the presence of lipid, a horizontal brain section was stained with luxol fast blue [Fig. 7(d)], which is a commonly used to observe myelin. Comparing the cerebellum of the mouse brain in Fig. 7(a) and 7(b) with 7(d) and 7(e), the cerebellar white matter structures match well with each other. Combined with the conclusion from Fig. 6, Fig. 7(d) tells us that the absorption difference between myelin and cytochrome sharpens PACT image contrast. A potential future study would focus on directly imaging lipid in the mouse brain by employing infrared light around 1200 and $1700 \mathrm{~nm}$, which are the $\mathrm{C}-\mathrm{H}$ bond absorption peaks. $^{57}$

We also studied the feasibility of imaging structures on nonperfused brains. To minimize the distortion of PA waves due to bone, we removed the skulls of a euthanized mouse, fully exposing the blood intact brain. Images acquired at different depths are shown in Fig. 8. It can be seen that both of the cortex vessels [Fig. 8(a)] and deep brain structure [Fig. 8(b) and 8(c)] are clearly resolved. Most of the cerebral structures, such as the neocortex, corpus callosum, hippocampus, hypothalamus, and ventricles can be identified [Fig. 8(c)]. Compared to Fig. 4(a), though the presence of hemoglobin decreases the structural contrast of the brain image, as shown in Video 4.

Nevertheless, the main hindrance to imaging the structure of the brain in vivo is the bone effect. Figure 3(b) shows that removing just the top skulls would not help us much. In fact, the top skulls removal created irregular bone boundaries, which further distorted the acoustic wave. Compared to the top skulls, the side skulls are thicker and have more complex structures. side bones have a stronger effect on the PA signal, because of our signal detection geometry (Fig. 1). To address this issue, there are two possible approaches. First, we can thin the skull to mitigate the acoustic distortion. Top-skull thinning is routinely applied in confocal or two-photon microscopy. ${ }^{58,59}$ Thinning the side skulls may require special attention to avoid loss of blood. Second, the acoustic properties of the head can be incorporated into PACT image reconstruction. This will require accurate measurement of the skull's geometry and acoustic properties, which can be achieved with other imaging modalities. For example, by using X-ray CT, the skull morphology and composition can be obtained and incorporated into a time-reversal-based reconstruction algorithm to correct for the skull distortion. ${ }^{60}$ Ultrasound tomography (UST) has also been integrated into PACT to measure the acoustic properties, which were then used to inform the PACT image reconstruction. ${ }^{61}$ With continuing advances in imaging techniques and reconstruction algorithms, we believe PACT will soon allow in vivo imaging of the deep brain in addition to the cortex.

In summary, we have experimentally demonstrated, for the first time, that PACT is able to provide label-free imaging of whole mouse brain structures in the absence of the side skulls, with MRM image quality. As a quantitative imaging modality, PACT can spectrally differentiate different chromophores (cytochromes and lipid) in brain tissue by virtue of its optical absorption contrast. As a deep imaging modality, PACT can resolve deep structures of the brain owing to its high-ultrasonic 


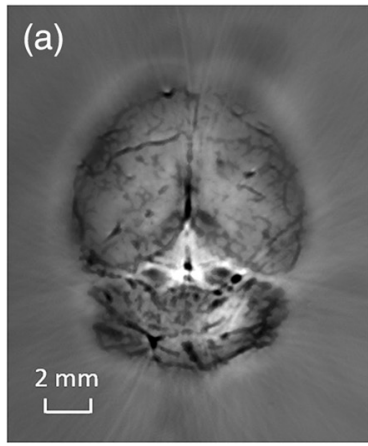

Depth: $0.3 \mathrm{~mm}$

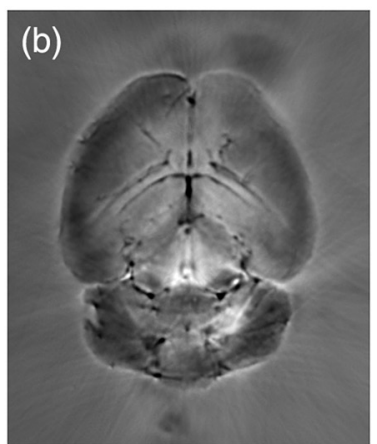

Depth: $2.1 \mathrm{~mm}$

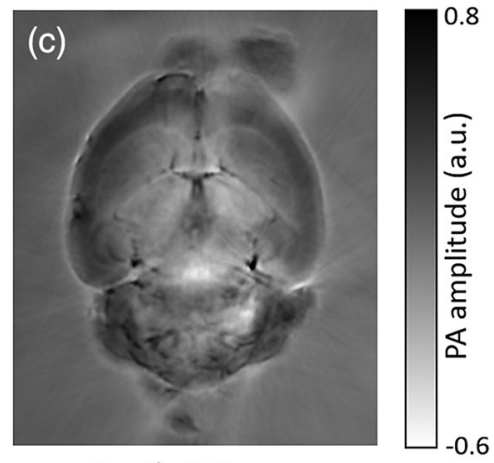

Depth: $2.9 \mathrm{~mm}$

Fig. 8 PACT image of a whole blood-perfused brain, showing both the vasculature and brain structure at depths of (a) $0.3 \mathrm{~mm}$, (b) $2.1 \mathrm{~mm}$, and (c) $2.9 \mathrm{~mm}$ (Video 4, MOV, $192 \mathrm{~KB}$ ) [URL: http://dx.doi.org/10 .1117/1.NPh.3.3.035001.4].

resolution. With these merits, we expect PACT to be applied to more brain structural and functional studies in the future.

\section{Acknowledgments}

The authors appreciate Prof. James Ballard's close reading of the manuscript, and thank Ernie Gonzales, Dr. Junjie Yao, Dr. Chi Zhang, and Chiye Li for their useful discussion and technical assistance. This work was sponsored in part by the National Institutes of Health (NIH) Grant Nos. DP1 EB016986 (NIH Director's Pioneer Award), R01 CA186567 (NIH Director's Transformative Research Award), U01 NS090579 (BRAIN Initiative), and R01 EB016963. L. V. Wang has a financial interest in Microphotoacoustics, Inc., which, however, did not support this work. L. V. Wang has a financial interest in Endra, Inc., and Microphotoacoustics, Inc., which, however, did not support this work. The other authors declare no competing financial interests.

\section{References}

1. J. D. Pollock, "Deep imaging technology needed for NIH BRAIN initiative," J. Biomed. Opt. 19(3), 030601 (2014).

2. N. Chuang et al., "An MRI-based atlas and database of the developing mouse brain," Neurolmage 54, 80-89 (2011).

3. D. N. Guilfoyle et al., "Functional connectivity fMRI in mouse brain at 7 T using isoflurane," J. Neurosc. Methods 214, 144-148 (2013).

4. J. Ellegood et al., "Neuroanatomical analysis of the BTBR mouse model of autism using magnetic resonance imaging and diffusion tensor imaging," Neurolmage 70, 288-300 (2013).

5. D. Wu et al., "Localized diffusion magnetic resonance micro-imaging of the live mouse brain," Neurolmage 91, 12-20 (2014).

6. F. Calamante et al., "Super-resolution track-density imaging studies of mouse brain: comparison to histology," NeuroImage 59, 286-296 (2012).

7. D. Wu et al., "In vivo high-resolution diffusion tensor imaging of the mouse brain," Neurolmage 83, 18-26 (2013).

8. Y. Jiang and G. A. Johnson, "Microscopic diffusion tensor atlas of the mouse brain," Neurolmage 56, 1235-1243 (2011).

9. R. Yuste and W. Denk, "Dendritic spines as basic functional units of neuronal integration," Nature 375, 682-684 (1995).

10. E. S. Boyden et al., "Millisecond-timescale, genetically targeted optical control of neural activity," Nat. Neurosci. 8, 1263-1268 (2005).

11. F. Zhang et al., "Multimodal fast optical interrogation of neural circuitry," Nature 446, 633-U634 (2007).

12. V. Gradinaru et al., "Optical deconstruction of parkinsonian neural circuitry," Science 324, 354-359 (2009).

13. T. E. Matthews et al., "In vivo and ex vivo epi-mode pump-probe imaging of melanin and microvasculature," Biomed. Opt. Express 2, 1576-1583 (2011).
14. C. W. Freudiger et al., "Label-free biomedical imaging with high sensitivity by stimulated Raman scattering microscopy," Science $\mathbf{3 2 2}$, 1857-1861 (2008)

15. L. Cai, N. Friedman, and X. S. Xie, "Stochastic protein expression in individual cells at the single molecule level," Nature 440, 358-362 (2006).

16. A. Y. Shih et al., "Two-photon microscopy as a tool to study blood flow and neurovascular coupling in the rodent brain," J. Cereb. Blood Flow Metab. 32, 1277-1309 (2012).

17. H. P. Lu, L. Y. Xun, and X. S. Xie, "Single-molecule enzymatic dynamics," Science 282, 1877-1882 (1998).

18. L. H. V. Wang and S. Hu, "Photoacoustic tomography: in vivo imaging from organelles to organs," Science 335, 1458-1462 (2012).

19. J. Xia, J. J. Yao, and L. H. V. Wang, "Photoacoustic tomography: principles and advances (invited review)," Prog. Electromagn. Res. 147, 22 (2014).

20. V. Ntziachristos et al., "Looking and listening to light: The evolution of whole-body photonic imaging," Nat. Biotechnol. 23, 313-320 (2005).

21. P. Beard, "Biomedical photoacoustic imaging," Interface Focus 1, 602-631 (2011)

22. D. Razansky et al., "Multispectral opto-acoustic tomography of deep-seated fluorescent proteins in vivo," Nat. Photonics 3, 412-417 (2009).

23. D. Razansky, A. Buehler, and V. Ntziachristos, "Volumetric real-time multispectral optoacoustic tomography of biomarkers," Nat. Protoc. 6, 1121-1129 (2011).

24. J. Xia et al., "Whole-body ring-shaped confocal photoacoustic computed tomography of small animals in vivo," J. Biomed. Opt. 17(5), 050503 (2012).

25. J. Gamelin et al., "A real-time photoacoustic tomography system for small animals," Opt. Express 17, 10489-10498 (2009).

26. X. D. Wang et al., "Noninvasive laser-induced photoacoustic tomography for structural and functional in vivo imaging of the brain," Nat. Biotechnol. 21, 803-806 (2003).

27. L.-D. Liao et al., "Imaging brain hemodynamic changes during rat forepaw electrical stimulation using functional photoacoustic microscopy," NeuroImage 52, 562-570 (2010).

28. J. Xia et al., "Three-dimensional photoacoustic tomography based on the focal-line concept," J. Biomed. Opt. 16(9), 090505 (2011).

29. M. H. Xu and L. H. V. Wang, "Universal back-projection algorithm for photoacoustic computed tomography," Phys. Rev. E 71, 7 (2005).

30. Q. Sheng et al., "Photoacoustic computed tomography without accurate ultrasonic transducer responses," Proc. SPIE 9323, 932313 (2015).

31. L. Wang, "Photoacoustic tomography," Scholarpedia 9(2), 10278 (2014).

32. Y. Jiang et al., "Blood oxygen flux estimation with a combined photoacoustic and high-frequency ultrasound microscopy system: a phantom study," J. Biomed. Opt. 17(3), 036012 (2012).

33. A. Ray et al., "Lifetime-based photoacoustic oxygen sensing in vivo," J. Biomed. Opt. 17(5), 057004 (2012).

34. L. Li et al., "Fully motorized optical-resolution photoacoustic microscopy," Opt. Lett. 39, 2117-2120 (2014). 
35. S. E. Forest and J. D. Simon, "Wavelength-dependent photoacoustic calorimetry study of melanin," Photochem. Photobiol. 68, 296-298 (1998).

36. J. A. Viator et al., "A comparative study of photoacoustic and reflectance methods for determination of epidermal melanin content," J. Investig. Dermatol. 122, 1432-1439 (2004).

37. M. Szakall et al., "On the pressure dependent sensitivity of a photoacoustic water vapor detector using active laser modulation control," Infrared Phys. Technol. 48, 192-201 (2006).

38. Z. Xu, Q. Zhu, and L. V. Wang, "In vivo photoacoustic tomography of mouse cerebral edema induced by cold injury," J. Biomed. Opt. 16(6), 066020 (2011).

39. T. J. Allen et al., "Spectroscopic photoacoustic imaging of lipid-rich plaques in the human aorta in the 740 to $1400 \mathrm{~nm}$ wavelength range," J. Biomed. Opt. 17(6), 061209 (2012).

40. B. Wang et al., "Intravascular photoacoustic imaging of lipid in atherosclerotic plaques in the presence of luminal blood," Opt. Lett. 37, 1244-1246 (2012).

41. P. Wang et al., "Mapping lipid and collagen by multispectral photoacoustic imaging of chemical bond vibration," J. Biomed. Opt. 17(9), 096010 (2012).

42. M. R. Bugs and M. L. Cornelio, "Analysis of the ethidium bromide bound to DNA by photoacoustic and FTIR spectroscopy," Photochem. Photobiol. 74, 512-520 (2001).

43. M. R. Bugs and M. L. Cornelio, "A new biophysics approach using photoacoustic spectroscopy to study the DNA-ethidium bromide interaction," Eur. Biophys. J. Biophys. Lett. 31, 232-240 (2002).

44. D. K. Yao et al., "In vivo label-free photoacoustic microscopy of cell nuclei by excitation of DNA and RNA," Opt. Lett. 35, 4139-4141 (2010).

45. C. Di Primo et al., "Origin of the photoacoustic signal in cytochrome p450(cam): Role of the Arg186-Asp251-Lys178 bifurcated salt bridge," Biochemistry 36, 112-118 (1997).

46. C. Zhang et al., "Label-free photoacoustic microscopy of cytochromes," J. Biomed. Opt. 18(2), 020504 (2013).

47. http://omlc.ogi.edu/spectra

48. http://www.ucl.ac.uk/medphys/research/borl/intro/spectra

49. G. S. Scott Brady, R. Wayne Albers, and D. Price, Basic Neurochemistry: Molecular, Cellular and Medical Aspects, 7th ed., p. 01803, Elsevier Academic Press, Burlington, Massachusetts (2006).

50. Y. Ma et al, "In vivo 3D digital atlas database of the adult C57BL/6J mouse brain by magnetic resonance microscopy," Front. Neuroanat. 2, 10 (2008).

51. J. C. Sutherland and K. P. Griffin, "Absorption-spectrum of DNA for wavelengths greater than 300-nm," Radiat. Res. 86, 399-410 (1981).

52. W. L. Lin et al., "An absolute measurement of brain water content using magnetic resonance imaging in two focal cerebral ischemic rat models," J. Cereb. Blood Flow Metab. 20, 37-44 (2000).

53. M. Picard and B. S. McEwen, "Mitochondria impact brain function and cognition," Proc. Natl. Acad. Sci. U. S. A. 111, 7-8 (2014).

54. A. V. Brusnichkin et al., "Ultrasensitive label-free photothermal imaging, spectral identification, and quantification of cytochrome c in mitochondria, live cells, and solutions," J. Biophotonics 3, 791-806 (2010).

55. M. Tanaka et al., "Connexin43 and bergmann glial gap junctions in cerebellar function," Front. Neurosci. 2, 225-233 (2008).

56. S. Kim et al., "Enhanced delineation of white matter structures of the fixed mouse brain using Gd-DTPA in microscopic MRI," NMR Biomed. 22, 303-309 (2009).

57. J. Hui et al., "Bond-selective photoacoustic imaging by converting molecular vibration into acoustic waves," Photoacoustics 4, 11-21 (2016).
58. D. F. Marker et al., "A thin-skull window technique for chronic twophoton in vivo imaging of murine microglia in models of neuroinflammation," J. Visualized Exp. (43), 2059 (2010).

59. G. Yang et al., "Thinned-skull cranial window technique for long-term imaging of the cortex in live mice," Nat. Protoc. 5, 201-208 (2010).

60. C. Huang et al., "Aberration correction for transcranial photoacoustic tomography of primates employing adjunct image data," J. Biomed. Opt. 17(6), 066016 (2012).

61. J. Xia et al., "Enhancement of photoacoustic tomography by ultrasonic computed tomography based on optical excitation of elements of a full-ring transducer array," Opt. Lett. 38, 3140-3143 (2013).

Lei $\mathbf{L i}$ received his $B S$ and $M S$ degrees from Harbin Institute of Technology, China, in 2010 and 2012, respectively. He is working as a graduate research assistant under the tutelage of Dr. Lihong Wang at Washington University. His research interests include PA microscopy and tomography, especially improving the PA imaging speed and applying it on brain functional and structural imaging.

Jun Xia received his PhD from the University of Toronto. Currently, he is a postdoctoral fellow at Washington University in St. Louis, under the mentorship of Dr. Lihong V. Wang. His research interests include the development of novel biomedical imaging techniques including PA, photothermal, and ultrasonic imaging. He has published more than 20 peer-reviewed journal articles in PA and photothermal research.

Guo Li received his BS degree in mechanical engineering and his MS degree in engineering physics from Tsinghua University. Currently, he is a graduate student at Washington University in St. Louis and a research assistant in Dr. Lihong V. Wang's laboratory. His research interests include high-frequency linear-array PA imaging.

Alejandro Garcia-Uribe received his MSc and PhD degrees in electrical engineering from the Department of Electrical and Computer Engineering, Texas A\&M University, College Station, Texas, USA. Currently, he is a research scientist at the Optical Imaging Laboratory, Department of Biomedical Engineering, Washington University in St. Louis. His research interests include biomedical optics, biomedical image analysis, microsensors, and digital signal processing.

Qiwei Sheng received his $\mathrm{PhD}$ in applied mathematical and computational sciences from the University of lowa. Currently, he is a research assistant at the Computational Bioimaging Laboratory, Washington University in St. Louis. His research interests include image reconstruction methods for PA and UST, numerical methods for partial differential equation, and inverse problems.

Mark A. Anastasio received his PhD from the University of Chicago. Currently, he is a professor of biomedical engineering at Washington University in St. Louis. His research interests include tomographic image reconstruction, imaging physics, and the development of novel-computed biomedical imaging systems. He has conducted extensive research in the fields of diffraction tomography, $x$-ray phase-contrast x-ray imaging, and PAT.

Lihong V. Wang received his PhD from Rice University, Houston, Texas, USA. Currently, he holds the Gene K. Beare distinguished professorship of biomedical engineering at Washington University in St. Louis. He has published 342 peer-reviewed journal articles and delivered 370 keynote, plenary, or invited talks. His Google Scholar h-index and citations have reached 81 and over 26,000, respectively. 